\title{
Desenvolvendo a Programação de Jogos Digitais no Ensino Médio: um Relato de Experiência Utilizando a Ferramenta Construct2
}

\author{
Thiago Reis da Silva ${ }^{1}$ \\ ${ }^{1}$ Instituto Federal de Educação, Ciência e Tecnologia do Maranhão - IFMA \\ Campus São João dos Patos - MA - Brasil \\ Grupo de Pesquisa em Ambientes Inteligentes, IoT e Sistemas Educacionais - GRAISE \\ thiago.reiseifma.edu.br
}

\begin{abstract}
In this article, we present an experience report with twenty high school students aiming to teach digital game programming. In order to be able to develop the games, the Construct 2 tool was used. The results show that it is possible to include the theme in the daily life of the schools in a way that arouses students' interest in technology in high school and also shows the acceptance of Construct 2 as a potential tool in the context of game programming learning.
\end{abstract}

Resumo. Neste artigo apresentamos um relato de experiência realizado com vinte alunos do ensino médio com o objetivo de ensinar programação de jogos digitais. Para que fosse possível o desenvolvimento dos jogos, a ferramenta Construct 2 foi utilizada. Os resultados alcançados mostram que é possível incluir a temática no cotidiano das escolas de maneira que desperte o interesse dos alunos pela tecnologia no ensino médio e, também, evidenciam a aceitação da Construct 2 como ferramenta potencial no contexto da aprendizagem de programação de jogos.

\section{Introdução}

Estudos apresentam que a demanda por profissionais para a área de computação vem aumentando a cada ano [Observatório Softex 2012]. Por outro lado, há uma tendência de queda na procura por cursos superiores na área e os índices de evasão em tais cursos estão entre os maiores [Barbosa, Ferreira e Costa 2014; Galdino, Silva Neto e Costa 2015]. Segundo o programa TI Maior - Programa estratégico de Software e Serviços de Tecnologia da Informação (TI) [2012-2015] ${ }^{1}$, as taxas de evasão em cursos de graduação do segmento de TI no país chegaram a 82\% em 2010. Nesta mesma pesquisa, identificou-se como um dos principais fatores desse problema é o baixo despertar vocacional dos estudantes da educação básica e do ensino profissional para as áreas tecnológicas.

A aprendizagem de programação, por exemplo, no ensino médio pode não apenas atrair profissionais com aptidão e talento para a computação, como também ajudar estudantes de outras áreas em sua educação ou em suas futuras profissões. Nunes (2011) exemplifica a situação dos advogados que, na medida em que leem um texto, usam o pensamento computacional para extrair dele fatos e regras que justifiquem um

${ }^{1}$ http://timaior.mcti.gov.br/ 
VI Congresso Brasileiro de Informática na Educação (CBIE 2017)

Anais dos Workshops do VI Congresso Brasileiro de Informática na Educação (WCBIE 2017)

parecer conclusivo. Outros procedimentos, como organizar processos, também podem ser apresentados de forma algorítmica.

Uma abordagem que vem sendo utilizada para tentar diminuir o declínio de interesse em cursos de computação e tentar alcançar os alunos de forma envolvente é a utilização de jogos [Jesus e Raabe 2010]. Os jogos são considerados uma poderosa ferramenta educacional e acredita-se que resultam em uma ampla gama de benefícios, aumentando a eficácia de aprendizagem, interesse, motivação e persistência [Silva, Aranha e Medeiros 2014].

Neste contexto, existem fatores que apontam a relevância da inserção do ensino de programação nas escolas. Scaico et. al. (2012) destacam dois fatores, o primeiro é que o ensino de programação permite o desenvolvimento de capacidades que contribuem para melhorar o raciocínio lógico dos estudantes. O segundo indica que tal proximidade tecnológica pode contribuir para acertar a visão e as atitudes dos estudantes com relação à finalidade e ao uso das tecnologias que habitualmente manuseiam.

Infelizmente, a maioria das escolas brasileiras ainda não está preparada para este desafio. A maioria das escolas brasileiras de ensino fundamental e médio não oferece nenhuma disciplina ou atividades que procurem ensinar noções básicas de programação de computadores [Batista et. al. 2015]. Mas isso vem mudando, como mostra as pesquisas de França e Tedesco (2015) e de diversas iniciativas, entre elas, a Iniciativa Computação na Escola ${ }^{2}$, o Programa Meninas Digitais ${ }^{3}$ e o Desafio Gamedu ${ }^{4}$.

Considerando a importância deste tema, este artigo apresenta os resultados de um estudo com alunos do ensino médio que promoveu o ensino de programação de jogos digitais. A aprendizagem de programação de jogos digitais foi facilitada pela ferramenta Construct $2^{5}$. Portanto, este estudo teve como objetivos: incentivar à concepção e o desenvolvimento de jogos digitais; incentivar a programação no ensino médio; e capacitar e desenvolver as habilidades dos alunos no desenvolvimento de jogos digitais através do uso da ferramenta de jogos Construct2.

Sendo assim, as próximas seções deste trabalho estão organizadas da seguinte maneira: a Seção 2 apresenta os trabalhos relacionados, a Seção 3 apresenta a ferramenta Construct2, enquanto que na Seção 4 descreve a metodologia utilizada. O planejamento do estudo é reportado na Seção 5. Os resultados são apresentados na Seção 6, e por fim, as considerações finais são tratadas na Seção 7.

\section{Trabalhos Relacionados}

Estudos envolvendo ensino de programação com alunos do ensino médio, com a temática dos jogos, já foram reportados por outros pesquisadores como as descritas a seguir.

\footnotetext{
${ }^{2}$ http://computacaonaescola.ufsc.br

${ }^{3}$ http://meninasdigitais.sbc.org.br

${ }^{4} \mathrm{http}: / /$ desafio.gamedu.net/

${ }^{5}$ https://www.scirra.com/
} 
Leal e Ferreira (2013) apresentam um estudo empírico com alunos do ensino médio na aprendizagem de programação de computadores com o uso de jogos. No estudo, ao desenvolverem os jogos, os estudantes fizeram as conexões com os padrões de programação no processo de ensino e aprendizagem de programação de computadores. Os resultados mostram uma melhoria na motivação e aprendizagem ao se aliar padrões de programação com jogos.

Al-Bow et. al., (2009) apresentam os resultados de um desafio de programação de jogos realizado por alunos e professores do ensino médio durante um mês. Os resultados do estudo indicam após o desafio um maior conhecimento de programação e um aumento da autoconfiança dos estudantes e professores com o tema.

Silva et. al., (2016) reporta um relato de experiência com alunos do ensino médio programando jogos com o Construct2. Os resultados do trabalho apontam que os alunos aprenderam conceitos relacionados à área da computação e conseguiram aplicar o conhecimento adquirido para desenvolver os jogos. Os resultados evidenciaram a aceitação do Construct 2 como ferramenta no apoio à aprendizagem de programação de jogos para este público. Em Silva et. al., (2015) é reportado os resultados de uma investigação de dois formatos de videoaulas de programação de jogos digitais para alunos do ensino médio. Os resultados despertaram um interesse inicial e motivacional dos alunos para essa área de conhecimento, pouco explorada na educação básica. $\mathrm{O}$ estudo também apresenta que os alunos têm interesse que esse conteúdo seja aplicado no currículo das escolas.

Doerschuk, Mann e Liu (2012) abordam uma academia de programação de jogos para alunos do ensino médio com o objetivo de envolver esses alunos com a área de computação explorando aplicações lúdicas. Os resultados demonstraram um aumento, tanto no conhecimento desses alunos, como no interesse deles para com a área de computação. Esse estudo indica que atividades práticas envolvendo programação de jogos não só podem ser eficazes no ensino de conceitos introdutórios de programação para estudantes do ensino médio, mas também podem ajudá-los a aumentar o seu interesse em computação e, em participação de cursos relacionados à área de TI. Em desfecho, convém lembrar que os estudos aqui destacados, de uma forma ou de outra, estão relacionados com o propósito deste estudo.

\section{A Ferramenta Construct2}

A Construct 2 é uma ferramenta de desenvolvimento de jogos desenvolvida pela Scirra. É uma ferramenta para criação de jogos em HyperText Markup Language (HTML5) que não requer conhecimento específico de uma linguagem de programação. $\mathrm{O}$ usuário só precisa arrastar e soltar itens na interface do programa e adicionar comportamentos a eles, não sendo necessário codificar, mas programar de forma visual [Andrade et. al. 2012]. Para esse estudo utilizamos a versão grátis do Construct2.

No Construct 2 o usuário dispõe os elementos do jogo e cria o enredo do mesmo, usando cliques do mouse, com a ação de arrastar e soltar os objetos no cenário principal [Medeiros, Silva e Aranha 2013]. A escolha dessa ferramenta ocorreu pela facilidade de 
utilização e pela baixa curva de aprendizagem e também pela mesma utilizar o conceito WYSIWYG (What You See Is What You Get - o que você vê é o que você tem), ou seja, o aluno consegue visualizar cada fase do jogo e enxergar como ficará o resultado final em tempo de desenvolvimento, sem a necessidade de fazer códigos. Outro ponto importante pela escolha do Construct 2 é devido a facilidade de implementar jogos para outras plataformas, diminuindo o tempo de implementação de jogos voltados para dispositivos móveis e para Web tendo em vista a utilização do HTML5.

\section{Metodologia}

A metodologia do estudo constitui-se da seguinte forma. Primeiramente foi realizado um convite aos alunos do primeiro ano do ensino médio do Instituto Federal de Educação Ciência e Tecnologia do Maranhão, Campus São Raimundo das Mangabeiras dos turnos manhã e tarde que tivesse interesse em participar de um estudo relacionado a aprendizagem de programação de jogos digitais. A seleção dos alunos foi realizada através de um convite. A pesquisa realizada foi exploratória, na qual foram utilizados para a análise os dados obtidos através dos formulários, observações e de satisfação dos alunos que participaram do projeto.

Dois jogos foram utilizados como primeira atividade de contatos dos estudantes com conceitos de programação. Foram utilizados os seguintes jogos que possuem complexidade de desenvolvimento similar: (i) o jogo Arkanoid é formado por uma bola, uma raquete e blocos coloridos, sendo o objetivo do jogo destruir com a bola todos os blocos coloridos, e (ii) o jogo nave tem como objetivo controlar uma nave para atirar e destruir naves inimigas que tentam eliminá-lo, ganhando pontos sempre que destrói uma nave inimiga.

Os estudantes foram divididos em duas turmas, cada um com 10 alunos que participaram do estudo em dias diferentes. A divisão dos alunos ocorreu de forma aleatória. Durante dois dias cada turma desenvolveu o jogo em 4 horas. Explicações gerais foram feitas antes do início do estudo. As eventuais dúvidas eram sanadas de forma que todos os alunos pudessem ouvir, para que um aluno não tivesse um determinado privilégio. Não houve explicações prévias sobre conceitos de programação de forma que o uso jogos representou um primeiro contato dos estudantes com o tema. $\mathrm{E}$ a partir deste uso as explicações eram fornecidas conforme as dúvidas dos alunos ocorresse.

\section{Planejamento}

Este estudo foi realizado em um laboratório com a participação de 20 alunos. Para isso, foi desenvolvido duas videoaulas, uma para o jogo arkanoid e outra para o jogo nave. Cada videoaula explicava conteúdos que o jogo possuía. O jogo arkanoid é composto por um conjunto de 14 pequenos vídeos, e o jogo Nave, por 13 pequenos vídeos. Cada vídeo com um tempo de duração que varia de 30 segundos a 6 minutos. Por exemplo, para montar cenário de um jogo, utiliza o comportamento Sprite da ferramenta 
VI Congresso Brasileiro de Informática na Educação (CBIE 2017)

Anais dos Workshops do VI Congresso Brasileiro de Informática na Educação (WCBIE 2017)

Construct2. O Sprite é utilizado para colocar objetos no jogo. O vídeo desse comportamento possui 3 minutos.

Cada conjunto de videoaula era composto pelos vídeos necessários para se desenvolver um jogo. Esse jogo possuía uma videoaula de apresentação e outros vídeos denominados de recursos que explicavam elementos da ferramenta de programação e de conceitos básicos de programação necessários para se desenvolver o jogo $\mathrm{e}$ funcionalidades específicas da ferramenta [Silva et. al. 2016].

O estudo foi realizado em três dias, cada dia com uma sessão de 4 horas. $\mathrm{O}$ primeiro dia teve como objetivo explicar como seria o estudo e apresenta uma visão geral do Construct2 e de como os alunos deveriam prosseguir durante $o$ desenvolvimento dos jogos. A principal preocupação era instruir os alunos a seguir o desenvolvimento corretamente, caso contrário, eles poderiam tentar explorar a ferramenta para outro propósito, que não fosse o foco do estudo. $\mathrm{O}$ segundo e terceiro dia foram utilizados para o desenvolvimento dos jogos, cada dia desenvolvendo um jogo diferente, de acordo com o determinado pela aleatorização (sorteio) e de forma monitorada pelo condutor e ao final de cada dia os alunos respondiam a um questionário.

A descrição geral do estudo é apresentada na Tabela 1. De modo indireto, objetivou-se também oferecer uma nova perspectiva aos alunos de que a programação pode ser algo divertido, interessante e desafiadora.

Tabela 1. Plane jamento do es tudo.

\begin{tabular}{|c|l|}
\hline Conteúdo & \multicolumn{1}{|c|}{ Descrição } \\
\hline Objetivo & $\begin{array}{l}\text { Incentivar à concepção e o desenvolvimento de jogos digitais; } \\
\text { incentivar a programação no ensino básico; capacitar e desenvolver as } \\
\text { habilidades dos alunos no desenvolvimento de jogos digitais através do } \\
\text { uso da ferramenta de jogos Construct2 }\end{array}$ \\
\hline Metodologia & $\begin{array}{l}\text { Aprendizagem através de descoberta da ferramenta Construct2, guiada } \\
\text { por desafios propostos pelas videoaulas de programação dos jogos }\end{array}$ \\
\hline Conteúdos & $\begin{array}{l}\text { Montar cenários, Movimentação entre Objetos, Pontuação do Jogo, } \\
\text { Variáveis, Colisão e Derrota do Jogo }\end{array}$ \\
\hline Local & Laboratório de Informática \\
\hline Participantes & 20 alunos \\
\hline Período & 3 dias \\
\hline
\end{tabular}

\subsection{Perfil dos Alunos}

O estudo foi realizado em um laboratório de informática com a participação de 20 estudantes. Esses participantes concordaram previamente em participar do estudo e assinaram um termo juntamente com os pesquisadores garantindo a confidencialidade da identidade deles no estudo. Os alunos tinham entre 14 a 17 anos, sendo a média de 15 anos. Desses 20 alunos, 14 foram homens e 6 mulheres, ou seja, $70 \%$ homens e $30 \%$ mulheres. 
Estes alunos também preencheram um formulário de caracterização com questões objetivas informando sobre sua experiência em temas relacionados ao estudo, como: (i) experiência em programação e (ii) conhecimento sobre a ferramenta Construct2. Desses alunos 8 tinha conhecimento básico sobre a linguagem de marcação HTML e apenas 1 tinha já tinha utilizado o Construct2 em outra oportunidade.

Sobre a experiência com jogos digitais, a maioria de ambos os grupos informou dedicar mais de 6 horas por semana ao uso de jogos. No que diz respeito ao gênero dos jogos preferidos dos alunos, os gêneros preferidos são: ação, aventura, estratégia e RPG.

\section{Resultados}

O estudo possibilitou aos alunos conhecerem noções básicas sobre programação de jogos digitais e o seu processo de criação. Sendo assim, para mensurar as impressões e opiniões dos estudantes a respeito do desenvolvimento dos jogos, analisamos os dados recolhidos dos alunos através de questionários e observações in loco. Todos os alunos o responderam os questionários de forma voluntária, não sendo obrigatória a participação dos mesmos.

Durante a criação do jogo Arkanoid, as dúvidas dos participantes se concentravam em como evitar que a bola não passasse pela barra e como fazer a bola voltar após tocar na barra. Para solucionar essa dúvida, os alunos assistiram novamente o vídeo do comportamento Solid do Construct2, esse comportamento faz com o que objeto não possa ser atravessado do layout definido para o jogo. Esse comportamento foi aplicado tanto na bola como na barra. Após rever o vídeo do comportamento todos conseguiram fazer o movimento da bola que desejavam.

Uma dúvida recorrente foi como fazer a bola apontar para um lugar aleatório ao tocar na barra. Para isso os alunos, tiveram que criar um evento de Colisão entre Objetos entre a barra e a bola.

No jogo da Nave, introduziu-se o conceito de variável, quando foi necessário criar a pontuação do jogo. A principal dúvida reportado nesse jogo era como fazer a derrota do jogo. Para esse caso os alunos usaram o Restart Layout que têm como função reiniciar cenários e eventos do jogo. Os recursos dos jogos, por exemplo, imagens e sons, foram disponibilizados para download, mas os alunos puderam desenhar seus próprios objetos, caso preferissem.

Através dos questionários os alunos avaliaram o estudo de forma geral. Indagados sobre sua motivação ao desenvolverem seus próprios jogos, $85 \%$ estavam muito motivados, $15 \%$ estavam motivados. Não foram reportados item como desmotivação no estudo. Desta forma, nota-se que os alunos estavam motivados.

Para investigar se foi estimulante, ou se o participante estava fazendo o estudo por fazer, avaliou-se o grau de interesse de cada aluno, os resultados foram: o acompanhamento do conteúdo do jogo foi ótimo para $65 \%$, para $35 \%$ foi bom e para $5 \%$ foi regular. No relacionamento do estudo com a formação escolar, $100 \%$ avaliaram que existe, principalmente com a matemática e física. Esse resultado corrobora com a 
afirmação de $95 \%$ dos alunos em ter uma disciplina de programação de jogos na escola, com pelos menos duas horas semanais.

Como resultados alcançados dos procedimentos utilizados no estudo, destaca-se a possibilidade de estimular o raciocínio lógico e a resolução de problemas de forma lúdica e dinâmica, através do desenvolvimento dos jogos, além do desenvolvimento de noções básicas de programação, como, tipos de dados, variáveis e estruturas condicionais. Essas habilidades podem ajudar os alunos em disciplinas como, matemática bem como em atividades presentes no seu cotidiano. É importante salientar, que os alunos desenvolveram habilidades de seleção e organização de informações necessárias para o desenvolvimento do jogo e se apropriaram dos recursos inerentes ao Construct2. A atividade de desenvolvimento do jogo, por caracteriza-se de natureza colaborativa, possibilitaram estimular a resolução de conflitos, o trabalho em equipe e a tomada de decisão para atingirem um objetivo comum, que nosso caso é o desenvolvimento integral do jogo.

No geral os resultados foram positivos, levando em consideração a avaliação dos conteúdos que foram compreendidos pelos alunos. Tal como também o julgamento da metodologia, que os agradou significativamente, pois os alunos a medida que assistiam as videoaulas já iniciava o desenvolvimento dos jogos. Portanto, os objetivos definidos ao realizar este trabalho foram alcançados. Os alunos demonstraram interesse em participar de novos estudos. Acreditamos que a vontade da maioria dos alunos em participar de um novo estudo se deu a partir da metodologia que foi utilizada durante as aulas e principalmente por desenvolverem seus próprios jogos.

Com relação as limitações do estudo, os alunos reportaram a necessidade de mais aulas práticas em laboratório com uma quantidade maior de horas e outros tipos de jogos para o desenvolvimento.

\subsection{Lições Aprendidas}

Inicialmente, alguns alunos demonstraram dificuldades básicas, como a diferença entre comportamentos da ferramenta utilizada e a elaboração dos passos para o desenvolvimento do jogo, visto que alguns alunos estavam concentrados no resultado final do jogo e não como chegar até esse resultado. Mas, conforme o andamento do estudo, essas dificuldades iniciais foram sendo superadas.

Os resultados alcançados evidenciaram também, os benefícios da tentativa e erro. Uma vez que o resultado esperado não era alcançado, os alunos poderiam recomeçar e repetir esse processo quantas vezes fossem necessárias para a conclusão daquela etapa do jogo. Depoimentos dos alunos corroboram essa afirmação: "Tem a vantagem de poder voltar quantas vezes quiser quando não entender". Outro resultado importante foi que os alunos puderam desenvolver seus jogos sem saber inicialmente de programação: "Interessante é um meio prático, diferente e dinâmico de se desenvolver um jogo e o mais legal de tudo é não precisamos ter nenhum conhecimento de programação". 
No estudo os alunos se submergiram nas atividades de aprendizagem envolvendo conhecimento e diversão relacionada ao desenvolvimento do jogo. Foi perceptível que a ideia que programação "é difícil" foi superada em partes, sobretudo, no desenvolvimento de algo interessante para os alunos (o jogo) sem conhecimento de linguagens de programação.

\subsection{Análise do Construct2}

Com relação a utilização do Construct2 como ferramenta para compreensão e desenvolvimento dos jogos, dentre os participantes, $100 \%$ reportaram interesse e afinidade com a ferramenta.

Ensinar programação de jogos digitais utilizando o Construct2 mostrou-se proveitoso. A execução em tempo real do comportamento desenvolvimento auxiliou os alunos a entender o que eles estavam implementando.

Os alunos ficaram concentrados e focados nas tarefas, mostrando motivação e satisfação em realizá-las. Durante o estudo, eles mostraram uma reação positiva em relação a aprendizagem da ferramenta, isso foi notório no desenvolvimento dos jogos. Também houve interesse em dar continuidade ao jogo e do uso da Construct2. O envolvimento com a ferramenta foi perceptível, muitos mencionaram que estavam praticando em casa, fazendo outros jogos.

No Construct2 a programação do jogo é feita visualmente, utilizando os conceitos de evento e ações, onde os eventos podem definir uma ou várias ações. $\mathrm{O}$ desenvolvimento rápido do jogo é um elemento chave da ferramenta, bastando o desenvolvedor criar um objeto, adicionar um comportamento, programar os eventos e ações do jogo tudo de forma visual para o jogo está funcional.

Dessa forma, os resultados mostram que a viabilidade do uso do Construct2 é recomendada para esse tipo de usuário, uma vez que os alunos vão aprender a criar seus jogos através de programação visual.

\section{Considerações finais}

Esse trabalho apresentou um relato de experiência de ensino de programação de jogos digitais utilizando a ferramenta Construct2 como facilitador. Em relação ao desenvolvimento dos jogos no ensino médio, observamos que a manipulação dos comportamentos da ferramenta consegue simplificar a execução de algoritmos e estruturas de programação.

De acordo com os resultados evidenciados, fica claro que a aplicação e o desenvolvimento de jogos pelos próprios alunos é um importante meio de auxílio ao processo de ensino e aprendizagem. Além desse modo de aprendizado fazendo com que os alunos solucionem o problema - o desenvolvimento do jogo - envolver alunos e professores em atividades que estimulam conhecimento sobre o pensamento computacional e uso de jogos digitais no ensino básico, pode servir como fator motivacional para os alunos aprenderem outros conteúdos. 
Diante dos resultados, destacamos que a diversão é um elemento importante, pois possibilita ampliar o engajamento e a motivação que são elementos fundamentais para facilitar a aprendizagem e o uso de jogos nesse processo motiva os alunos, isso foi perceptível durante a execução do estudo.

Outro ponto importante foi a facilidade de utilização da ferramenta Construct2, focada no desenvolvimento de jogos. Para a utilização desta ferramenta o aluno não precisa ter conhecimento de programação para o desenvolvimento dos jogos. Isto viabilizou os aspectos que envolve o desenvolvimento do jogo, como, as imagens, os comportamentos e os cenários, a serem utilizados no jogo.

A contribuição deste trabalho vai de encontro com o pensamento atual e cada vez mais aceito de que a computação é sim importante em todos os níveis escolares e sempre que possível deve ser inserido no currículo das escolas. E o uso de jogos digitais facilita a inclusão da computação nas escolas. Ainda, este trabalho visa estimular outros trabalhos futuros em escolas públicas e/ou privadas que tenham como objetivos estimular a programação, e com isso poder modificar a visão que a computação é "chata" e "difícil", contribuindo assim, para atrair mais alunos para a computação.

Neste caso, considerando os resultados iniciais já alcançados, e pensando em perpetuá-los e melhorá-los, iremos ampliar o campo de estudo, inserindo outras ferramentas de programação de jogos e também incluindo linguagens de programação.

\section{Agradecimentos}

O autor agradece o Instituto Federal de Educação, Ciência e Tecnologia do Maranhão IFMA, Campus São João dos Patos pelo apoio parcial a esta pesquisa.

\section{Referências}

Al-Bow, M.; Austin, D.; Edgington, J.; Fajardo, R.; Fishburn, J.; Lara, C.; Leutenegger, S.; Meyer, S. (2009) "Using Game Creation for Teaching Computer Programming to High School Students and Teachers". In: ACM Conference on Innovation and Technology in Computer Science Education - ITiCSE, p.104-108.

Andrade, M.; Almeida, E.; Silva, C.; Melo, M. T.; Oliveira, T.; Falcão, F.; Araújo, A. (2012) "Coletando: Desenvolvimento de um jogo para o ensino-aprendizagem no campo da Educação Ambiental". In: Simpósio Brasileiro de Jogos e Entretenimento Digital - SBGAMES, p.188-191.

Batista, W. P.; Chalegre, H. S.; Sena, J. P. S.; Santos, A. H. M.; Santos, D. M. B.; Rodrigues, C. A.; Bittencourt, R. A. (2015) "Oficinas de Aprendizagem de Programação em uma Escola Pública através do Ambiente Scratch”. In: XXIII Workshop sobre Educação em Computação - WEI.

Barbosa, L. S.; Fernandes, T. C. B.; Campos, A. M. C. (2011) "Takkou: Uma Ferramenta Proposta ao Ensino de Algoritmos". In: XIX Workshop sobre Educação em Computação - WEI, Natal, Brasil, 2011. 
VI Congresso Brasileiro de Informática na Educação (CBIE 2017)

Anais dos Workshops do VI Congresso Brasileiro de Informática na Educação (WCBIE 2017)

Doerschuk, P.; Mann, J.; Liu, J. (2012) “An INSPIRED game programming academy for high school students". In: IEEE Frontiers in Education Conference - FIE.

França, R. S.; Tedesco, P. C. A. R. (2015) "Desafios e oportunidades ao ensino do pensamento computacional na educação básica no Brasil”. In: Workshop de Ensino em Pensamento Computacional, Algoritmos e Programação - WalProg.

Galdino, C. B. T.; Silva Neto, S. R.; Costa, E. B. (2015) "KidCoder: Uma Proposta de Ensino de Programação de forma Lúdica". In: XXVI Simpósio Brasileiro de Informática na Educação - SBIE, p.687-691, 2015.

Jesus, E. A.; Raabe, A. L. A. (2009) "Interpretações da Taxonomia de Bloom no Contexto da Programação Introdutória”. In: XX Simpósio Brasileiro de Informática na Educação - SBIE, Florianópolis - SC, Brasil.

Leal, A. V. A.; Ferreira, D. J. (2013) “Teaching computer programming based on patterns with activities and collaborative games using concrete materials for high school students". In: 43rd Annual Conference Frontiers in Education - FIE, p.16041610 .

Medeiros, T. J.; Silva, T. R.; Aranha, E. H. S. (2013) "Ensino de programação utilizando jogos digitais: uma revisão sistemática da literatura". In: Revista Novas Tecnologias na Educação - RENOTE, v.11, n.3.

Nunes, D. J. Ciência da Computação na Educação Básica. 2011. Disponível em: <http://www.adufrgs.org.br/artigos/ciencia-da-computacao-na-educacao-basica/>. Acessado em: set. de 2015.

Observatório Softex (2012). "Software e Serviços de TI: a indústria Brasileira em Perspectiva". v.2.

Scaico, P. D.; Marques, D. L.; Melo, L. A.; Azevedo, M. A.; Mendes Neto, S. V.; Oliveira, A.; Alves Júnior, J.; Labanca, M.; Scaico, A. (2012) "Um jogo para o ensino de programação em Python baseado na taxonomia de Bloom”. In: Workshop de Educação em Computação - WEI.

Silva, T. R.; Medeiros, T. J.; Aranha, E. H. S. (2014) "Jogos Digitais para Ensino e Aprendizagem de Programação: uma Revisão Sistemática da Literatura”. In: XXV Simpósio Brasileiro de Informática na Educação - SBIE, p.692-701.

Silva, T. R.; Aranha, E. H. S.; Oliveira, W.; Fernandes, K. T.; Lucena, M. J. N. R. (2015) "Investigando dois formatos de videoaulas de programação de jogos digitais para alunos do ensino médio". In: XXI Workshop de Informática na Escola - WIE, p.187- 196.

Silva, T. R.; Aranha, E. H. S.; Santos, F. G.; Tavares, K. F. (2016) "Um relato de experiência da aplicação de videoaulas de programação de jogos digitais para alunos da educação básica". In: XXII Workshop sobre Educação em Computação - WEI, Uberlândia - MG. 